\title{
The complex dynamics of a stochastic toxic-phytoplankton-zooplankton model
}

Feng Rao*

\section{"Correspondence:}

raofeng2002@163.com

College of Sciences, Nanjing

University of Technology, Puzhu

Road, Nanjing, 211816, China

\section{Springer}

\begin{abstract}
In this paper, an analytical study of a toxin-producing phytoplankton-zooplankton model with stochastic perturbation is performed. By constructing suitable Lyapunov functions, we investigate the global stability of a positive equilibrium and give the condition of the existence of Hopf bifurcation for the deterministic plankton model. Under the perturbation of environmental noise, there is a globally positive solution to the stochastic model and it is stochastically ultimately bounded. In addition, the stochastic model is stochastically permanent under some conditions. A series of numerical simulations to illustrate these mathematical findings are presented.
\end{abstract}

Keywords: toxic-phytoplankton-zooplankton model; stochastic perturbation; stability

\section{Introduction}

Phytoplankton are primary producers as the base of the aquatic food web, floating freely near the surfaces of all aquatic environments. Zooplankton are the animals in the plankton community and feed on other phytoplankton [1]. Phytoplankton and zooplankton are subject to water movements to a large extent. They act as the basis of all food chains and webs in aquatic systems and play an important role in the ecology of the ocean $[2,3]$. In the last two decades, there has been a global increase in harmful plankton blooms in aquatic ecosystem [4-6]. Bandyopadhyay et al. [7] pointed out that a broad classification of harmful plankton species distinguishes two groups: one is the toxin producers, which can contaminate seafood or kill fish, and the other is the high-biomass producers, which can cause anoxia and indiscriminate mortalities of marine life after reaching dense concentrations. The toxin-producing phytoplankton play an important role on the growth of the zooplankton population. And because of phytoplankton and zooplankton universal existence and importance, understanding of the dynamical behaviors of interacting species will continue to be a predominant topic. In recent years, a great deal of attention has been paid towards in toxin-producing plankton blooms and a lot of its extensions from several researchers [2, 3, 7-14].

In the real world, population dynamics is inevitably subjected to environmental noise, which is an important component in an ecosystem. Most natural phenomena do not follow strictly deterministic laws, but rather oscillate randomly about some average values. So that the population density never attains a fixed value with the advancement of time $[15,16]$. The basic mechanism and factors of population growth like resources and vital rates - birth, death, immigration, and emigration - change non-deterministically due

○2014 Rao; licensee Springer. This is an Open Access article distributed under the terms of the Creative Commons Attribution License (http://creativecommons.org/licenses/by/2.0), which permits unrestricted use, distribution, and reproduction in any medium, provided the original work is properly cited. 
to continuous fluctuations in the environment (e.g. variation in intensity of sunlight, temperature, water level, etc.) [17]. Recent advances in stochastic differential equations enable a lot of authors to introduce randomness into a deterministic model of physical phenomena to reveal the effect of environmental variability, whether it is a random noise in the system of differential equations or environmental fluctuations in parameters, see, e.g. [18-26]. Of them, Beddington and May [18] studied harvesting natural populations in a randomly fluctuating environment. In [19], Mao showed that the noise cannot only have a destabilising effect but can also have a stabilising effect in the control theory. The growth of populations in a random environment subjected to variable effort fishing policies was studied in [20]. Braumann [21] generalized the previous results [20] to density-dependent positive noise intensities of general form so that they also become independent from the way environmental fluctuations affect population growth rates. Liu et al. [23] presented a spatial version of the phytoplankton-zooplankton model that includes some important factors such as external periodic forces, noise and diffusion processes. These important results reveal the significant effects of the environmental noise on some models.

To our knowledge, a toxin-producing phytoplankton-zooplankton model with a Holling type-II functional response has deserved a lot of attention, but mainly in deterministic case. The research on the dynamical behavior of the toxic-phytoplankton-zooplankton model with Holling type-II functional response under environmental noise seems rare. Based on the discussion above, in this paper, we focus on dynamical properties of a toxinproducing plankton model with Holling type-II functional response under stochastic perturbation. The organization of this paper is as follows. In the next section, a stochastic toxic-phytoplankton-zooplankton model is established. Firstly, we give a general survey of the stability analysis of a positive equilibrium of the model without noise. Then, we concentrate our attention on the stochastic version of the toxin producing phytoplanktonzooplankton model and discuss the existence of global positive solution, stochastic boundedness, and the global asymptotic stability of the stochastic model. In Section 3, we give some numerical examples and make a comparative analysis of the stability of the model system within deterministic and stochastic environments. Finally, we give a concluding remark section.

\section{Model and dynamical analysis}

Referring to the work of $[2,3,8]$, they introduced and analyzed toxin producing phytoplankton-zooplankton systems. Chattopadhayay et al. [8] observed the effect of toxinproducing plankton on zooplankton from the field-collected samples and mathematical modeling. In [2], Saha and Bandyopadhyay considered a toxin producing phytoplanktonzooplankton model in which the toxic liberation by phytoplankton species follows a discrete time variation. Lv et al. [3] proposed and investigated a phytoplankton-zooplankton model with harvesting. Based on their studies, in this paper, we consider a stochastic form of toxin-producing plankton model and intend to analyze the model with the assumption that $f(P)$ and $g(P)$ are described by same type of function, namely, Holling type-II function. With this Holling type-II functional response and logistic phytoplankton growth, the deterministic toxin producing phytoplankton-zooplankton model is

$$
\left\{\begin{array}{l}
\frac{\mathrm{d} P}{\mathrm{~d} t}=r\left(P\left(1-\frac{P}{K}\right)-a f(P) Z\right)=r P\left(1-\frac{P}{K}-\frac{a Z}{m+P}\right), \\
\frac{\mathrm{d} Z}{\mathrm{~d} t}=b Z(f(P)-d-c g(P))=b Z\left(\frac{(1-c) P}{m+P}-d\right) .
\end{array}\right.
$$


In the above model, $P=P(t)$ is the density of toxin producing phytoplankton population and $Z=Z(t)$ is the density of zooplankton population at any instant of time $t . f(P)$ represents the functional response for the grazing of phytoplankton by zooplankton and $g(P)$ descries the distribution of toxin substance which ultimately contributes to the death of zooplankton populations.

Based on model (1), we assume that random fluctuations in the environment would display themselves mainly as fluctuations in the intrinsic growth rates of the toxin producing phytoplankton and the zooplankton, then replace parameters $r$ and $b$ by

$$
r \rightarrow r+\sigma_{1} \dot{B}_{1}(t), \quad b \rightarrow b+\sigma_{2} \dot{B}_{2}(t)
$$

Hence, we are mainly interested in the dynamics of the stochastic model which takes the following form:

$$
\left\{\begin{array}{l}
\mathrm{d} P=P\left(1-\frac{P}{K}-\frac{a Z}{m+P}\right)\left(r \mathrm{~d} t+\sigma_{1} \mathrm{~d} B_{1}(t)\right), \\
\mathrm{d} Z=Z\left(\frac{(1-c) P}{m+P}-d\right)\left(b \mathrm{~d} t+\sigma_{2} \mathrm{~d} B_{2}(t)\right),
\end{array}\right.
$$

where $\sigma_{1}^{2}$ and $\sigma_{2}^{2}$ are known as the intensities of environmental noise. $\dot{B}_{i}(t)(i=1,2)$ is a standard white noise, that is, $B_{i}(t)(i=1,2)$ independent Brownian motion defined in a complete probability space $(\Omega, \mathcal{F}, \mathbf{P})$ with a filtration $\left\{\mathcal{F}_{t}\right\}_{t \in R_{+}}$satisfying the usual conditions (i.e., it is right continuous and increasing while $\mathcal{F}_{0}$ contains all $\mathbf{P}$-null sets) [27]. Parameters $K, m, a, c, d$ are positive constants, where $K$ is the environmental carrying capacity of toxin-producing phytoplankton population, $m$ the half saturation constant for a Holling type-II functional response, $a$ the maximum uptake rate for zooplankton species, $c$ the rate of toxic substances produced by per unit biomass of phytoplankton and $d$ the natural death rate of zooplankton.

\subsection{Dynamical analysis of model (1)}

Model (1) has three equilibria in the positive quadrant:

(i) $E_{0}=(0,0)$ (total extinct) is a saddle point.

(ii) $E_{1}=(K, 0)$ (extinct of the zooplankton, or phytoplankton-only) is a stable node if $K(1-c-d)<m d$ and a saddle point if $K(1-c-d)>m d$.

(iii) $E^{*}=\left(P^{*}, Z^{*}\right)$ (coexistence of the phytoplankton and zooplankton) is a positive interior equilibrium, where

$$
P^{*}=\frac{m d}{1-c-d}, \quad Z^{*}=\frac{m(1-c)(K(1-c-d)-m d)}{a K(1-c-d)^{2}},
$$

with $c+d<1$ and $K(1-c-d)>m d$.

From the biological point of view, studying the stability behavior of positive equilibrium $E^{*}=\left(P^{*}, Z^{*}\right)$ is of real interest. The Jacobian matrix corresponding to $E^{*}=\left(P^{*}, Z^{*}\right)$ of model (1) is

$$
\begin{aligned}
J & =J\left(E^{*}\right)=\left(\begin{array}{cc}
\frac{d r(K(1-c-d)-m(1-c+d))}{K(1-c)(1-c-d)} & -\frac{a d r}{1-c} \\
\frac{b(K(1-c-d)-m d)}{a K} & 0
\end{array}\right) \\
& =\left(\begin{array}{ll}
J_{11} & J_{12} \\
J_{21} & J_{22}
\end{array}\right) .
\end{aligned}
$$


The characteristic equation is given by

$$
\lambda^{2}-\operatorname{tr}(J) \lambda+\operatorname{det}(J)=0
$$

where

$$
\begin{aligned}
& \operatorname{tr}(J)=\frac{d r(K(1-c-d)-m(1-c+d))}{K(1-c)(1-c-d)}, \\
& \operatorname{det}(J)=\frac{b d r(K(1-c-d)-m d)}{K(1-c)}
\end{aligned}
$$

By the Roth-Hurwitz criterion, we have a sufficient condition for the local stability of $E^{*}=$ $\left(P^{*}, Z^{*}\right)$ of model (1).

Theorem 2.1 If $m d<K(1-c-d)<m(1-c+d)$ holds, then the positive equilibrium $E^{*}=$ $\left(P^{*}, Z^{*}\right)$ of model (1) is locally asymptotically stable.

Theorem 2.2 If $K<m\left(m+P^{*}\right) /\left(a Z^{*}\right)$ and $b m(1-c) /\left(\operatorname{ar}\left(m+P^{*}\right)\right)<P / Z$ hold, then the positive equilibrium $E^{*}=\left(P^{*}, Z^{*}\right)$ of model (1) is globally asymptotically stable in the interior of the first octant.

Proof Define a Lyapunov function

$$
V(P, Z)=\left(P-P^{*}\right)^{2}+\left(Z-Z^{*}\right)^{2}
$$

which is nonnegative and $V(P, Z)=0$ if and only if $(P, Z)=\left(P^{*}, Z^{*}\right)$. The function satisfies $\partial V / \partial P=2\left(P-P^{*}\right), \partial V / \partial Z=2\left(Z-Z^{*}\right)$ and it is easy to see that $\left(P^{*}, Z^{*}\right)$ is the only extremum and the global minimum of the function in the positive octant $R_{+}^{2}$. The time derivative of $V$ along the solutions of model (1) is

$$
\frac{\mathrm{d} V}{\mathrm{~d} t}=2\left(P-P^{*}\right) \frac{\mathrm{d} P}{\mathrm{~d} t}+2\left(Z-Z^{*}\right) \frac{\mathrm{d} Z}{\mathrm{~d} t}
$$

Substituting the expressions of $\mathrm{d} P / \mathrm{d} t$ and $\mathrm{d} Z / \mathrm{d} t$ from model (1), we obtain

$$
\frac{\mathrm{d} V}{\mathrm{~d} t}=r\left(P-P^{*}\right) P\left(1-\frac{P}{K}-\frac{a Z}{m+P}\right)+b\left(Z-Z^{*}\right) Z\left(\frac{(1-c) P}{m+P}-d\right) .
$$

Using the fact that

$$
1-\frac{P^{*}}{K}-\frac{a Z^{*}}{m+P^{*}}=0, \quad \frac{(1-c) P^{*}}{m+P^{*}}-d=0,
$$

equation (4) can be re-written as

$$
\begin{aligned}
\frac{\mathrm{d} V}{\mathrm{~d} t}= & -2 r P\left(\frac{1}{K}-\frac{a Z^{*}}{(m+P)\left(m+P^{*}\right)}\right)\left(P-P^{*}\right)^{2} \\
& -\left(\frac{2 a r P}{m+P}-\frac{2 b m(1-c) Z}{(m+P)\left(m+P^{*}\right)}\right)\left(P-P^{*}\right)\left(Z-Z^{*}\right) .
\end{aligned}
$$


Figure 1 Phase portrait of model (1) with $K=0.6, r$ $=4, m=0.5, a=0.9, b=0.7, c=0.1, d=0.2$. The horizontal axis is the phytoplankton population $P$ and the vertical axis is the zooplankton population $Z$. $E_{0}=(0,0)$ and $E_{1}=(0.6,0)$ are saddle points, the equilibrium point $E^{*}=(0.1429,0.5442)$ is globally asymptotically stable.

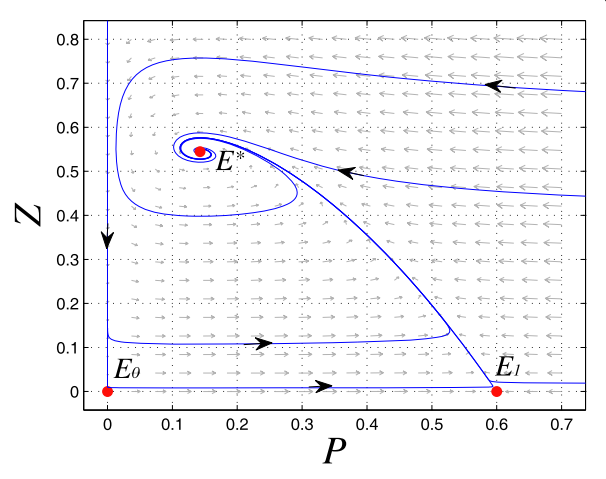

If the conditions $m\left(m+P^{*}\right) /\left(a Z^{*}\right)>K$ and $P / Z>b m(1-c) /\left(a r\left(m+P^{*}\right)\right)$ hold, then $\mathrm{d} V / \mathrm{d} t \leq$ 0 in $R_{+}^{2}$, where the equality holds only at the equilibrium point $\left(P^{*}, Z^{*}\right)$. Hence the equilibrium $E^{*}=\left(P^{*}, Z^{*}\right)$ is globally asymptotically stable.

When choosing the values of parameters $K=0.6, r=4, m=0.5, a=0.9, b=0.7, c=0.1$, $d=0.2$ for model $(1), E_{0}=(0,0)$ and $E_{1}=(0.6,0)$ are saddle points, the positive equilibrium point $\left(P^{*}, Z^{*}\right)=(0.1429,0.5442)$ exists. By Theorem $2.2, E^{*}=\left(P^{*}, Z^{*}\right)$ is globally asymptotically stable. The trajectory of the phytoplankton and zooplankton population of model $(1)$ is shown in Figure 1 , which shows $E^{*}=(0.1429,0.5442)$ is stable.

Referring to [28], we study the Hopf bifurcation of model (1), which is space-independent. It breaks the temporal symmetry of a system and gives rise to oscillations that are uniform in space and periodic in time. Here, taking $K$ as the bifurcation parameter, we have the following theorem.

Theorem 2.3 If $c+d<1$ holds, then model (1) undergoes Hopf bifurcation around the positive equilibrium point $E^{*}=\left(P^{*}, Z^{*}\right)$. The Hopf bifurcation occurs at its critical value $K=K_{H}=m(1-c+d) /(1-c-d)$.

Proof The Hopf bifurcation occurs if and only if there exists a $K=K_{H}$ such that

(i) $[\operatorname{tr}(J)]_{K=K_{H}}=0$;

(ii) $[\operatorname{det}(J)]_{K=K_{H}}=\frac{b d r(1-c-d)}{1-c+d}>0$;

(iii) when $E^{*}=\left(P^{*}, Z^{*}\right)$ exists, the characteristic equation is $\lambda^{2}+[\operatorname{det}(J)]_{K=K_{H}}=0$, whose roots are purely imaginary;

(iv) $\frac{\mathrm{d}}{\mathrm{d} K}[\operatorname{tr}(J)]_{K=K_{H}}=\frac{d r(1-c-d)}{m(1-c)(1-c+d)} \neq 0$.

Therefore, all the conditions of Hopf bifurcation theorem are satisfied, thus there exists a small amplitude periodic solution near $E^{*}=\left(P^{*}, Z^{*}\right)$. This completes the proof.

Figure 2 depicts time-series plots and phase portraits for model (1) under different carrying capacity $K$ values and other parameters are the same as in Figure 1 . In Figure 2(A), choosing $K=0.7<K_{H}=0.7857$, one can see that the positive equilibrium $\left(P^{*}, Z^{*}\right)=(0.1429,0.5685)$ is asymptotically stable point. In Figure $2(\mathrm{~B})$, increasing $K$ to $K_{H}=0.7857$, a stable limit cycle occurs which indicates that toxin-producing phytoplankton coexists with zooplankton with oscillator balance behavior. As $K$ is further increased, in Figure $2(\mathrm{C})$ with $K=1.0>K_{H}$, the interior equilibrium $\left(P^{*}, Z^{*}\right)=(0.1429,0.6122)$ is unstable and loses its stability as $K$ passes through its critical value $K_{H}=0.7857$. 
(A)
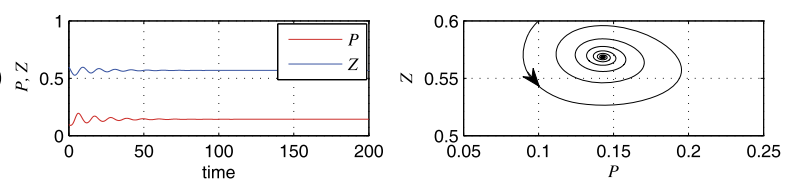

(B)
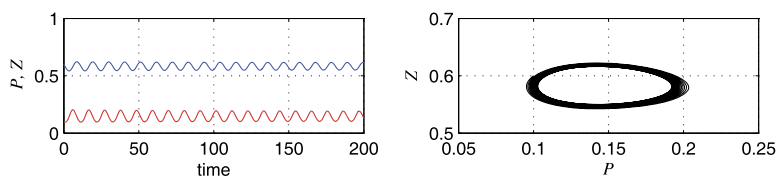

(C)
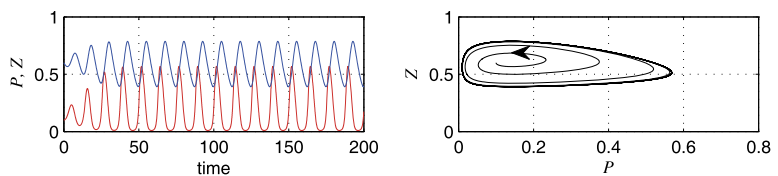

Figure 2 (Left) Time-series plots; (Right) phase portraits for model (1) with $r=4, m=0.5, a=0.9$, $\boldsymbol{b}=\mathbf{0 . 7}, \boldsymbol{c}=\mathbf{0 . 1}, \boldsymbol{d}=\mathbf{0 . 2}$ and initial condition $\left(\boldsymbol{P}_{\mathbf{0}}, \boldsymbol{Z}_{\mathbf{0}}\right)=(\mathbf{0 . 1}, \mathbf{0 . 6})$. (A) $K=0.7<K_{H}=0.7857 ;(\mathbf{B}) K=K_{H}$, a limit cycle occurs; (C) $K=1.0>K_{H}$.

\subsection{Dynamical analysis of model (2)}

In this subsection, we consider the stochastic model (2) as an Itô stochastic differential system of the type

$$
\mathrm{d} U(t)=F(U(t)) \mathrm{d} t+G(U(t)) \mathrm{d} B(t), \quad U_{t 0}=U_{0},
$$

where

$$
\begin{aligned}
& U(t)=\left(\begin{array}{c}
P(t) \\
Z(t)
\end{array}\right), \quad B(t)=\left(\begin{array}{c}
B_{1}(t) \\
B_{2}(t)
\end{array}\right), \quad F=\left(\begin{array}{c}
r P\left(1-\frac{P}{K}-\frac{a Z}{m+P}\right) \\
b Z\left(\frac{(1-c) P}{m+P}-d\right)
\end{array}\right), \\
& G=\left(\begin{array}{cc}
\sigma_{1} P\left(1-\frac{P}{K}-\frac{a Z}{m+P}\right) & 0 \\
0 & \sigma_{2} Z\left(\frac{(1-c) P}{m+P}-d\right)
\end{array}\right),
\end{aligned}
$$

and the solution $U(t)(t>0)$ is an Itô process.

Here we give the following auxiliary statements which are introduced in [27]. Denote by $C^{1,2}\left(\left[t_{0}, \infty\right] \times R^{2} ; R_{+}\right)$the family of all nonnegative functions $V(t, U)$ defined on $\left[t_{0}, \infty\right] \times$ $R^{2}$ such that they are continuously once differentiable in $t$ and twice in $U$. The diffusion operator $\mathbf{L}$ of the above equation (5) is defined by the formula

$$
\mathbf{L}=\frac{\partial}{\partial t}+\sum_{i=1}^{2} F_{i}(t, U) \frac{\partial}{\partial U_{i}}+\frac{1}{2} \sum_{i, j=1}^{2}\left[G^{T}(t, U) G(t, U)\right]_{i j} \frac{\partial^{2}}{\partial U_{i} \partial U_{i}} .
$$

If $\mathbf{L}$ acts on a function $V \in C^{1,2}\left(\left[t_{0}, \infty\right] \times R^{2} ; R_{+}\right)$, then

$$
\mathbf{L} V(t, U)=V_{t}(t, U)+V_{U}(t, U) F(t, U)+\frac{1}{2} \operatorname{tr}\left[G^{T}(t, U) V_{U U}(t, U) G(t, U)\right]
$$

For a stochastic differential equation, in order to have a unique global solution (that is, no explosion in a finite time) for any given initial value, the coefficients of the equation are generally required to satisfy the linear growth condition and local Lipschitz condition [27]. 
To show that model (2) has a positive global solution, let us firstly prove that the model has a positive local solution by making a change of variables.

Theorem 2.4 There is a unique local solution $(P(t), Z(t))$ for $t \in\left[0, \tau_{e}\right)$ to model (2) almost surely for the initial value $P_{0}>0, Z_{0}>0$, where $\tau_{e}$ is the explosion time.

Proof Set $u(t)=\ln P(t), v(t)=\ln Z(t)$; we consider the equations

$$
\left\{\begin{aligned}
\mathrm{d} u= & {\left[r\left(1-\frac{\mathrm{e}^{u}}{K}-\frac{a \mathrm{e}^{\nu}}{m+\mathrm{e}^{u}}\right)-\frac{\sigma_{1}^{2}}{2}\left(1-\frac{\mathrm{e}^{u}}{K}-\frac{a \mathrm{e}^{\nu}}{m+\mathrm{e}^{u}}\right)^{2}\right] \mathrm{d} t } \\
& +\sigma_{1}\left(1-\frac{\mathrm{e}^{u}}{K}-\frac{a \mathrm{e}^{v}}{m+\mathrm{e}^{u}}\right) \mathrm{d} B_{1}(t), \\
\mathrm{d} v= & {\left[b\left(\frac{(1-c) \mathrm{e}^{u}}{m+\mathrm{e}^{u}}-d\right)-\frac{\sigma_{2}^{2}}{2}\left(\frac{(1-c) e^{u}}{m+\mathrm{e}^{u}}-d\right)^{2}\right] \mathrm{d} t } \\
& +\sigma_{2}\left(\frac{(1-c) \mathrm{e}^{u}}{m+\mathrm{e}^{u}}-d\right) \mathrm{d} B_{2}(t), \\
u(0)= & \ln P_{0}, \quad v(0)=\ln Z_{0},
\end{aligned}\right.
$$

on $t \geq 0$. The coefficients of (6) satisfy the local Lipschitz condition and there is a unique local solution $(u(t), v(t))$ on $\left[0, \tau_{e}\right)$. By Itô's formula, we can see that $P(t)=\mathrm{e}^{u(t)}, Z(t)=\mathrm{e}^{v(t)}$ is the unique positive local solution to model (2) with initial value $P_{0}>0, Z_{0}>0$.

In the following, we show this solution is global, i.e., $\tau_{e}=\infty$

Theorem 2.5 For model (2) and any given initial value $\left(P_{0}, Z_{0}\right) \in R_{+}^{2}$, there is a unique solution $(P(t), Z(t))$ on $t \geq 0$ and the solution will remain in $R_{+}^{2}$ almost surely.

Proof Let $n_{0}>0$ be sufficiently large for $P_{0}$ and $Z_{0}$ lying within the interval $\left[1 / n_{0}, n_{0}\right]$. For each integer $n \geq n_{0}$, define the stopping times

$$
\tau_{n}=\inf \left\{t \in\left[0, \tau_{e}\right]: P(t) \notin\left(\frac{1}{n}, n\right) \text { or } Z(t) \notin\left(\frac{1}{n}, n\right)\right\},
$$

where we set $\inf \emptyset=\infty$ ( $\emptyset$ represents the empty set). $\tau_{n}$ is increasing as $n \rightarrow \infty$.

Let

$$
\tau_{\infty}=\lim _{n \rightarrow \infty} \tau_{n}
$$

then $\tau_{\infty} \leq \tau_{e}$ a.s. Now, we need to show $\tau_{\infty}=\infty$ a.s. If this statement is violated, there exist constants $T>0$ and $\varepsilon \in(0,1)$ such that

$$
\mathbf{P}\left\{\tau_{\infty} \leq T\right\}>\varepsilon .
$$

Then, there is an integer $n_{1} \geq n_{0}$ such that

$$
\mathbf{P}\left\{\tau_{n} \leq T\right\} \geq \varepsilon, \quad n \geq n_{1} .
$$

Define a $C^{2}$-function $V: R_{+}^{2} \rightarrow R_{+}$by

$$
V(P, Z)=\sqrt{P}+\sqrt{Z}-(\ln \sqrt{P}+\ln \sqrt{Z})-2,
$$


which is a nonnegative function. If $(P(t), Z(t)) \in R_{+}^{2}$, by using Itồs formula, we compute

$$
\begin{aligned}
& \mathrm{d} V(P, Z)= \frac{r}{2}(\sqrt{P}-1)\left(1-\frac{P}{K}-\frac{a Z}{m+P}\right)+\frac{b}{2}(\sqrt{Z}-1)\left(\frac{(1-c) P}{m+P}-d\right) \\
&+\frac{\sigma_{1}^{2}}{8}(2-\sqrt{P})\left(1-\frac{P}{K}-\frac{a Z}{m+P}\right)^{2} \\
&\left.+\frac{\sigma_{2}^{2}}{8}(2-\sqrt{Z})\left(\frac{(1-c) P}{m+P}-d\right)\right] \mathrm{d} t \\
&+\frac{\sigma_{1}}{2}(\sqrt{P}-1)\left(1-\frac{P}{K}-\frac{a Z}{m+P}\right) \mathrm{d} B_{1} \\
&+\frac{\sigma_{2}}{2}(\sqrt{Z}-1)\left(\frac{(1-c) P}{m+P}-d\right) \mathrm{d} B_{2} \\
& \leq \frac{r}{2}\left(\sqrt{P}+\frac{P}{K}-\frac{\sqrt{P^{3}}}{K}\right)+\frac{b c P}{2(m+P)}+\frac{\sigma_{2}^{2}(1-c)^{2} P^{2}}{4(m+P)^{2}} \\
&\left.+\frac{\sigma_{1}^{2}}{8}\left(2+\frac{2 P^{2}}{K^{2}}+\frac{2 \sqrt{P^{3}}}{K}-\frac{\sqrt{P^{5}}}{K^{2}}\right)\right] \mathrm{d} t \\
&+\left[\frac{a r Z}{2 m}+\frac{b d}{2}(1-\sqrt{Z})+\frac{\sigma_{1}^{2}}{8}\left(\frac{4 a Z}{K}+\frac{2 a^{2} Z^{2}}{m^{2}}+\frac{a Z}{\sqrt{m}}\right)\right. \\
&\left.+\frac{\sigma_{2}^{2}}{8}\left(2 d^{2}+2 d(1-c) \sqrt{Z}-d^{2} \sqrt{Z}\right)\right] \mathrm{d} t \\
&+\frac{\sigma_{1}}{2}(\sqrt{P}-1)\left(1-\frac{P}{K}-\frac{a Z}{m+P}\right) \mathrm{d} B_{1} \\
&+\frac{\sigma_{2}}{2}(\sqrt{Z}-1)\left(\frac{(1-c) P}{m+P}-d\right) \mathrm{d} B_{2} \\
& \leq\left(M_{1}+M_{2}\right) \mathrm{d} t+\frac{\sigma_{1}}{2}(\sqrt{P}-1)\left(1-\frac{P}{K}-\frac{a Z}{m+P}\right) \mathrm{d} B_{1} \\
&
\end{aligned}
$$

where $M_{1}, M_{2}$ are positive constants. Integrating both sides of the above inequality from 0 to $\tau_{n} \wedge T$ and then taking the expectations lead to

$$
\mathbf{E} V\left(P\left(\tau_{n} \wedge T\right), Z\left(\tau_{n} \wedge T\right)\right) \leq V\left(P_{0}, Z_{0}\right)+\left(M_{1}+M_{2}\right) T
$$

Set $\Omega_{n}=\left\{\tau_{n} \leq T\right\}$ and by inequality (7), we get $\mathbf{P}\left(\Omega_{n}\right) \geq \varepsilon$. For every $\omega \in \Omega_{n}$, there is some $i$ such that $x_{i}\left(\tau_{n}, \omega\right)$ equals either $n$ or $1 / n$ for $i=1,2$, hence $V\left(P\left(\tau_{n}, \omega\right), Z\left(\tau_{n}, \omega\right)\right)$ is no less than $\min \{(\sqrt{n}-\ln \sqrt{n}-1),(1 / \sqrt{n}+\ln \sqrt{n}-1)\}$. Then we obtain

$$
\begin{aligned}
V\left(P_{0}, Z_{0}\right)+\left(M_{1}+M_{2}\right) T & \geq \mathbf{E}\left[1_{\Omega_{n}}(\omega) V\left(P\left(\tau_{n}\right), Z\left(\tau_{n}\right)\right)\right] \\
& \geq \varepsilon \min \left\{(\sqrt{n}-\ln \sqrt{n}-1),\left(\frac{1}{\sqrt{n}}+\ln \sqrt{n}-1\right)\right\},
\end{aligned}
$$

where $1_{\Omega_{n}}$ is the indicator function of $\Omega_{n}$. Letting $n \rightarrow \infty$ leads to the contradiction $\infty=$ $V\left(P_{0}, Z_{0}\right)+\left(M_{1}+M_{2}\right) T<\infty$. This completes the proof. 
From Theorem 2.5, model (2) has a positive global solution. By constructing some Lyapunov functions, we analyze the stability of the positive equilibrium of the stochastic model.

Theorem 2.6 When $\Theta=-b m K(1-c) /\left(a\left(\sigma_{1}^{2} P^{*}-r K\right)\left(m+P^{*}\right)\right)$ and the conditions $r K / P^{*}<$ $\sigma_{1}^{2}$, and

$$
\begin{aligned}
& a \sigma_{2}^{2} Z^{*}(1-c)\left(\sigma_{1}^{2} P^{*}-r K\right) \\
& \quad<b m K\left(m+P^{*}\right)\left(\frac{a Z^{*}\left(2 m r\left(m+P^{*}\right)+a \sigma_{1}^{2} P^{*} Z^{*}\right)}{m^{2}\left(m+P^{*}\right)^{2}}+\frac{\sigma_{1}^{2} P^{*}-2 r K}{K^{2}}\right)
\end{aligned}
$$

hold, then for any initial value $\left(P_{0}, Z_{0}\right)$ the equilibrium point $\left(P^{*}, Z^{*}\right)$ of model (2) has the property

$$
\lim _{t \rightarrow \infty} P(t)=P^{*}, \quad \lim _{t \rightarrow \infty} Z(t)=Z^{*}
$$

almost surely.

Proof By the stability theory of stochastic differential equations [27], we only need to establish a Lyapunov function $V(U)$ satisfying $\mathbf{L} V(U) \leq 0$ and the identity holds if and only if $U=U^{*}$, where $U=U(t)$ is the solution of the stochastic differential equation (5) and $U^{*}$ is the equilibrium position of (5).

Define Lyapunov functions

$$
V_{1}(P)=P-P^{*}-P^{*} \ln \frac{P}{P^{*}}, \quad V_{2}(Z)=Z-Z^{*}-Z^{*} \ln \frac{Z}{Z^{*}} .
$$

Referring to the work of Mao et al. [29], the nonnegativity of these functions can be seen from that $x-1-\log x \geq 0$ on $x>0$. If $(P(t), Z(t)) \in R_{+}^{2}$, applying Itô's formula leads to

$$
\begin{aligned}
\mathbf{L} V_{1}= & r\left(P-P^{*}\right)\left(1-\frac{P}{K}-\frac{a Z}{m+P}\right)+\frac{\sigma_{1}^{2} P^{*}}{2}\left(1-\frac{P}{K}-\frac{a Z}{m+P}\right)^{2} \\
\leq & \left(\frac{a r Z^{*}}{m\left(m+P^{*}\right)}-\frac{r}{K}+\frac{a^{2} \sigma_{1}^{2} P^{*} Z^{* 2}}{2 m^{2}\left(m+P^{*}\right)^{2}}+\frac{\sigma_{1}^{2} P^{*}}{2 K^{2}}\right)\left(P-P^{*}\right)^{2} \\
& +\frac{a^{2} \sigma_{1}^{2} P^{*}}{2 m^{2}}\left(Z-Z^{*}\right)^{2}+\frac{a\left(\sigma_{1}^{2} P^{*}-r K\right)}{K(m+P)}\left(P-P^{*}\right)\left(Z-Z^{*}\right) \\
& +\frac{a^{2} \sigma_{1}^{2} P^{*} Z^{*}}{m^{2}\left(m+P^{*}\right)}\left|P-P^{*}\right|\left|Z-Z^{*}\right|, \\
\mathbf{L} V_{2}= & b\left(Z-Z^{*}\right)\left(\frac{(1-c) P}{m+P}-d\right)+\frac{\sigma_{2}^{2} Z^{*}}{2}\left(\frac{(1-c) P}{m+P}-d\right)^{2} \\
\leq & \frac{b m(1-c)}{(m+P)\left(m+P^{*}\right)}\left(P-P^{*}\right)\left(Z-Z^{*}\right)+\frac{\sigma_{2}^{2} Z^{*}(1-c)^{2}}{2\left(m+P^{*}\right)^{2}}\left(P-P^{*}\right)^{2} .
\end{aligned}
$$

Let $V(P, Z)=\Theta V_{1}(P)+V_{2}(Z)$; when we choose

$$
\Theta=-\frac{b m K(1-c)}{a\left(\sigma_{1}^{2} P^{*}-r K\right)\left(m+P^{*}\right)},
$$


then

$$
\begin{aligned}
\mathbf{L} V(P, Z)= & \mathbf{L} V_{1}(P)+\mathbf{L} V_{2}(Z) \\
= & \left\{\Theta \left[r\left(\frac{a Z^{*}}{\left(m+P^{*}\right)(m+P)}-\frac{1}{K}\right)\right.\right. \\
& \left.+\frac{\sigma_{1}^{2} P^{*}}{2}\left(\frac{a Z^{*}}{\left(m+P^{*}\right)(m+P)}-\frac{1}{K}\right)^{2}\right] \\
& \left.+\frac{m^{2} \sigma_{2}^{2} Z^{*}(1-c)^{2}}{2(m+P)^{2}\left(m+P^{*}\right)^{2}}\right\}\left(P-P^{*}\right)^{2}+\frac{\Theta a^{2} \sigma_{1}^{2} P^{*}}{2(m+P)^{2}}\left(Z-Z^{*}\right)^{2} \\
& -\left\{\Theta\left[\frac{a r}{m+P}+\frac{a \sigma_{1}^{2} P^{*}}{m+P}\left(\frac{a Z^{*}}{\left(m+P^{*}\right)(m+P)}-\frac{1}{K}\right)\right]\right. \\
& \left.-\frac{b m(1-c)}{(m+P)\left(m+P^{*}\right)}\right\}\left(P-P^{*}\right)\left(Z-Z^{*}\right) \\
\leq & -\frac{b m K(1-c)}{a\left(\sigma_{1}^{2} P^{*}-r K\right)\left(m+P^{*}\right)}\left(\frac{2 a r m Z^{*}\left(m+P^{*}\right)+a^{2} \sigma_{1}^{2} P^{*} Z^{* 2}}{2 m^{2}\left(m+P^{*}\right)^{2}}\right. \\
& \left.\left.+\frac{\sigma_{1}^{2} P^{*}-2 r K}{2 K^{2}}\right)+\frac{\sigma_{2}^{2} Z^{*}(1-c)^{2}}{2\left(m+P^{*}\right)^{2}}\right]\left(P-P^{*}\right)^{2} \\
& -\frac{b K a^{2} \sigma_{1}^{2}(1-c) P^{*}}{2 a m\left(\sigma_{1}^{2} P^{*}-r K\right)\left(m+P^{*}\right)}\left(Z-Z^{*}\right)^{2} \\
& -\frac{b K a^{2} \sigma_{1}^{2}(1-c) P^{*} Z^{*}}{a m\left(\sigma_{1}^{2} P^{*}-r K\right)\left(m+P^{*}\right)^{2}}\left|P-P^{*}\right|\left|Z-Z^{*}\right| .
\end{aligned}
$$

When $r K / P^{*}<\sigma_{1}^{2}$ and

$$
\begin{aligned}
& b m K\left(m+P^{*}\right)\left(\frac{a Z^{*}\left(2 m r\left(m+P^{*}\right)+a \sigma_{1}^{2} P^{*} Z^{*}\right)}{m^{2}\left(m+P^{*}\right)^{2}}+\frac{\sigma_{1}^{2} P^{*}-2 r K}{K^{2}}\right) \\
& >a \sigma_{2}^{2} Z^{*}(1-c)\left(\sigma_{1}^{2} P^{*}-r K\right),
\end{aligned}
$$

then we see that the above inequality implies $\mathbf{L} V(P, Z)<0$ along all trajectories in the first quadrant except $E^{*}=\left(P^{*}, Z^{*}\right)$. Hence, the theorem holds.

This theorem shows that when environmental noises satisfy some conditions, the unique solution of model (2) in $R_{+}^{2}$ is a stochastically asymptotically stable. That's to say, both species under the effect of noises can coexist in stable conditions and eventually tend to the equilibrium sate.

Theorem 2.5 shows that the solution to model (2) will remain in $R_{+}^{2}$. The property lets us continue to discuss how the solution varies in $R_{+}^{2}$ in more detail. We first present the definition of stochastic ultimate boundedness [30,31], one of the important topics in population dynamics and defined as follows.

Definition 2.1 The solution $(P(t), Z(t))$ of model (2) is said to be stochastically ultimately bounded, if, for any $\varepsilon \in(0,1)$, there is a positive constant $\delta=\delta(\varepsilon)$, such that for any initial value $\left(P_{0}, Z_{0}\right) \in R_{+}^{2}$, the solution $(P(t), Z(t))$ to model (2) has the property that

$$
\limsup _{t \rightarrow \infty} \mathbf{P}\{|(P(t), Z(t))|>\delta\}<\varepsilon .
$$


Theorem 2.7 The solution of model (2) is stochastically ultimately bounded for any initial value $\left(P_{0}, Z_{0}\right) \in R_{+}^{2}$.

Proof From Theorem 2.5, the solution will remain in $R_{+}^{2}$ for all $t \geq 0$ almost surely. Set a function $V_{1}=\mathrm{e}^{t} P^{\theta}$ for $(P, Z) \in R_{+}^{2}$ and $\theta>0$. By Itô's formula we obtain

$$
\begin{aligned}
\mathrm{d} V_{1}= & \mathrm{e}^{t} P^{\theta}\left[1+r \theta\left(1-\frac{P}{K}-\frac{a Z}{m+P}\right)+\frac{\sigma_{1}^{2} \theta(\theta-1)}{2}\left(1-\frac{P}{K}-\frac{a Z}{m+P}\right)^{2}\right] \mathrm{d} t \\
& +\sigma_{1} \theta \mathrm{e}^{t} P^{\theta}\left(1-\frac{P}{K}-\frac{a Z}{m+P}\right) \mathrm{d} B_{1} \\
\leq & C_{1} \mathrm{e}^{t} \mathrm{~d} t+\sigma_{1} \theta \mathrm{e}^{t} P^{\theta}\left(1-\frac{P}{K}-\frac{a Z}{m+P}\right) \mathrm{d} B_{1},
\end{aligned}
$$

then $\mathrm{e}^{t} \mathbf{E} P^{\theta}-\mathbf{E} P_{0}^{\theta} \leq C_{1} \mathrm{e}^{t}$. So we get

$$
\limsup _{t \rightarrow \infty} \mathbf{E} P^{\theta} \leq C_{1}<+\infty \text {. }
$$

Define $V_{2}=\mathrm{e}^{t} Z^{\theta}$, then

$$
\begin{aligned}
\mathrm{d} V_{2}= & \mathrm{e}^{t} Z^{\theta}\left[1+b \theta\left(\frac{(1-c) P}{m+P}-d\right)+\frac{\sigma_{2}^{2} \theta(\theta-1)}{2}\left(\frac{(1-c) P}{m+P}-d\right)^{2}\right] \mathrm{d} t \\
& +\sigma_{2} \theta \mathrm{e}^{t} Z^{\theta}\left(\frac{(1-c) P}{m+P}-d\right) \mathrm{d} B_{2} \\
\leq & C_{2} \mathrm{e}^{t} \mathrm{~d} t+\sigma_{2} \theta \mathrm{e}^{t} Z^{\theta}\left(\frac{(1-c) P}{m+P}-d\right) \mathrm{d} B_{2},
\end{aligned}
$$

and we also have

$$
\limsup _{t \rightarrow \infty} \mathbf{E} Z^{\theta} \leq C_{2}<+\infty
$$

Therefore, we obtain

$$
\limsup _{t \rightarrow \infty} \mathbf{E}\left(P^{2}(t)+Z^{2}(t)\right)^{\frac{\theta}{2}} \leq 2^{\frac{\theta}{2}}\left(C_{1}+C_{2}\right)<+\infty,
$$

and we have the required assertion by taking the Chebyshev inequality.

Generally speaking, the non-explosion property, the existence and the uniqueness of the solution are not enough, but the property of permanence is more desirable since it means long time survival in a population dynamics. Now, the definition of stochastic permanence [30-32] will be given below.

Definition 2.2 The solution $(P(t), Z(t))$ of model (2) is said to be stochastically permanent, if, for any $\varepsilon \in(0,1)$, there exists a pair of positive constants $\delta=\delta(\varepsilon)$ and $\chi=\chi(\varepsilon)$ such that for any initial value $\left(P_{0}, Z_{0}\right) \in R_{+}^{2}$, the solution $(P(t), Z(t))$ to model (2) has the properties that

$$
\liminf _{t \rightarrow \infty} \mathbf{P}\{|(P(t), Z(t))| \geq \delta\} \geq 1-\varepsilon, \quad \liminf _{t \rightarrow \infty} \mathbf{P}\{|(P(t), Z(t))| \leq \chi\} \geq 1-\varepsilon .
$$


Theorem 2.8 For any initial value $\left(P_{0}, Z_{0}\right) \in R_{+}^{2}$, the solution $(P(t), Z(t))$ satisfies

$$
\limsup _{t \rightarrow \infty} \mathbf{E}\left[\left(P^{2}+Z^{2}\right)^{-\frac{\theta}{2}}\right] \leq \frac{2^{\theta} C}{k}
$$

where $C$ is a positive constant and $\theta, k$ are arbitrary positive constants satisfying

$$
\theta \min \{r, b(1-c)\}>\frac{\theta(\theta+1)}{2} \max \left\{\sigma_{1}^{2}, \sigma_{2}^{2}\right\}+k
$$

Proof Set a function

$$
V(P, Z)=\frac{1}{P+Z}
$$

for $(P(t), Z(t)) \in R_{+}^{2}$, by Itô's formula, we have

$$
\begin{aligned}
\mathrm{d} V= & -V^{2}\left[r P\left(1-\frac{P}{K}-\frac{a Z}{m+P}\right)+b Z\left(\frac{(1-c) P}{m+P}-d\right)\right] \mathrm{d} t \\
& +V^{3}\left[\sigma_{1}^{2} P^{2}\left(1-\frac{P}{K}-\frac{a Z}{m+P}\right)^{2}+\sigma_{2}^{2} Z^{2}\left(\frac{(1-c) P}{m+P}-d\right)^{2}\right] \mathrm{d} t \\
& -V^{2}\left[\sigma_{1} P\left(1-\frac{P}{K}-\frac{a Z}{m+P}\right) \mathrm{d} B_{1}+\sigma_{2} Z\left(\frac{(1-c) P}{m+P}-d\right) \mathrm{d} B_{2}\right] .
\end{aligned}
$$

Then choosing a positive constant $\theta$ and using Itô's formula, we can get

$$
\begin{aligned}
& \mathbf{L}(1+V)^{\theta} \\
& =\theta(1+V)^{\theta-1}\left\{-V^{2}\left[r P\left(1-\frac{P}{K}-\frac{a Z}{m+P}\right)+b Z\left(\frac{(1-c) P}{m+P}-d\right)\right]\right. \\
& \left.\quad+V^{3}\left[\sigma_{1}^{2} P^{2}\left(1-\frac{P}{K}-\frac{a Z}{m+P}\right)^{2}+\sigma_{2}^{2} Z^{2}\left(\frac{(1-c) P}{m+P}-d\right)^{2}\right]\right\} \\
& \quad+\frac{\theta(\theta-1)}{2} V^{4}(1+V)^{\theta-2}\left[\sigma_{1}^{2} P^{2}\left(1-\frac{P}{K}-\frac{a Z}{m+P}\right)^{2}+\sigma_{2}^{2} Z^{2}\left(\frac{(1-c) P}{m+P}-d\right)^{2}\right]
\end{aligned}
$$

Let $k>0$ be sufficiently small, so that it satisfies (11), then

$$
\begin{aligned}
& \mathbf{L e}^{k t}(1+V)^{\theta} \\
& =\mathrm{e}^{k t}(1+V)^{\theta-2}\left\{k(1+V)^{2}-\theta V^{2}(1+V)\left[r P\left(1-\frac{P}{K}-\frac{a Z}{m+P}\right)+b Z\left(\frac{(1-c) P}{m+P}-d\right)\right]\right. \\
& \quad+\theta(1+V) V^{3}\left[\sigma_{1}^{2} P^{2}\left(1-\frac{P}{K}-\frac{a Z}{m+P}\right)^{2}+\sigma_{2}^{2} Z^{2}\left(\frac{(1-c) P}{m+P}-d\right)^{2}\right] \\
& \left.\quad+\frac{\theta(\theta-1)}{2} V^{4}\left[\sigma_{1}^{2} P^{2}\left(1-\frac{P}{K}-\frac{a Z}{m+P}\right)^{2}+\sigma_{2}^{2} Z^{2}\left(\frac{(1-c) P}{m+P}-d\right)^{2}\right]\right\} .
\end{aligned}
$$

We are motivated by reference [31] and the following inequalities:

$$
V^{3}\left(\sigma_{1}^{2} P^{2}+\sigma_{2}^{2} Z^{2}\right) \leq \max \left\{\sigma_{1}^{2}, \sigma_{2}^{2}\right\} V
$$


Therefore, we obtain

$$
\begin{aligned}
\mathbf{L e}^{k t}(1+V)^{\theta} \leq & \mathrm{e}^{k t}(1+V)^{\theta-2}\left[k+\theta \max \left\{\frac{r}{K}, a r+b d\right\}\right. \\
& +\left(2 k+\theta \max \left\{\frac{r}{K}, a r+b d\right\}-\theta \min \{r, b(1-c)\}+\theta \max \left\{\sigma_{1}^{2}, \sigma_{2}^{2}\right\}\right) V \\
& \left.-\left(\theta \min \{r, b(1-c)\}-\frac{\theta(\theta+1)}{2} \max \left\{\sigma_{1}^{2}, \sigma_{2}^{2}\right\}-k\right) V^{2}\right]
\end{aligned}
$$

There exists a positive constant $C$ such that $\mathbf{L e}^{k t}(1+V)^{\theta} \leq C \mathrm{e}^{k t}$; then

$$
\mathbf{E}\left[\mathrm{e}^{k t}(1+V)^{\theta}\right] \leq(1+V(0))^{\theta}+\frac{C}{k}\left(\mathrm{e}^{k t}-1\right) .
$$

So we can get

$$
\limsup _{t \rightarrow \infty} \mathbf{E} V^{\theta}(t) \leq \limsup _{t \rightarrow \infty} \mathbf{E}(1+V(t))^{\theta} \leq \frac{C}{k}
$$

In addition, we know that $(P+Z)^{\theta} \leq 2^{\theta}\left(P^{2}+Z^{2}\right)^{\frac{\theta}{2}}$, consequently,

$$
\limsup _{t \rightarrow \infty} \mathbf{E}\left[\frac{1}{\left(P^{2}+Z^{2}\right)^{\frac{\theta}{2}}}\right] \leq 2^{\theta} \limsup _{t \rightarrow \infty} \mathbf{E} V^{\theta}(t) \leq \frac{2^{\theta} C}{k} .
$$

The proof is complete.

Based on the results of Theorems 2.7, 2.8 and the Chebyshev inequality, we can obtain the following theorem.

Theorem 2.9 Assume that $\max \left\{\sigma_{1}^{2}, \sigma_{2}^{2}\right\}<2 \min \{r, b(1-c)\}$, the solution of model (2) is stochastically permanent.

In the above discussion, we show that under certain conditions, the stochastic model (2) is ultimately bounded and stochastically permanent. Here, we will show that if the noise is sufficiently large, the solution to the stochastic model will become extinct. In other words, the following theorem reveals the important fact that the environmental noise may make the population extinct.

Theorem 2.10 The solution $(P(t), Z(t))$ of model (2) with initial value $\left(P_{0}, Z_{0}\right) \in R_{+}^{2}$ has the following properties:

$$
\limsup _{t \rightarrow \infty} \frac{\ln P}{t} \leq r-\frac{\sigma_{1}^{2}}{2}, \quad \limsup _{t \rightarrow \infty} \frac{\ln Z}{t} \leq b-b d-\frac{\sigma_{2}^{2}}{2} \quad \text { a.s. }
$$

Proof It follows from model (2) that

$$
\left\{\begin{array}{l}
\mathrm{d} P \leq r P\left(1-\frac{P}{K}-\frac{a Z}{m+P}\right) \mathrm{d} t+\sigma_{1} P \mathrm{~d} B_{1}(t) \\
\mathrm{d} Z \leq b Z\left(\frac{(1-c) P}{m+P}-d\right) \mathrm{d} t+\sigma_{2} Z \mathrm{~d} B_{2}(t) .
\end{array}\right.
$$


Set

$$
\left\{\begin{array}{l}
\mathrm{d} \bar{P}=r \bar{P}\left(1-\frac{\bar{P}}{K}-\frac{a \bar{Z}}{m+\bar{P}}\right) \mathrm{d} t+\sigma_{1} \bar{P} \mathrm{~d} B_{1}(t), \\
\mathrm{d} \bar{Z}=b \bar{Z}\left(\frac{(1-c) \bar{P}}{m+\bar{P}}-d\right) \mathrm{d} t+\sigma_{2} \bar{Z} \mathrm{~d} B_{2}(t),
\end{array}\right.
$$

where $(\bar{P}(t), \bar{Z}(t))$ is a solution of model (14) with initial value $\bar{P}_{0}>0$ and $\bar{Z}_{0}>0$. By the comparison theorem for stochastic differential equations, it is easy to find

$$
P(t) \leq \bar{P}(t), \quad Z(t) \leq \bar{Z}(t), \quad \text { a.s. } t \in[0, \infty]
$$

For model (14), define the Lyapunov functions $\ln \bar{P}$ and $\ln \bar{Z}$, respectively, then by the Itô formula, we have

$$
\begin{aligned}
& \ln \bar{P}=\ln \bar{P}_{0}+\left(r-\frac{\sigma_{1}^{2}}{2}\right) t-\frac{r}{K} \int_{0}^{t} \bar{P}(s) \mathrm{d} s-a r \int_{0}^{t} \frac{\bar{Z}(s)}{m+\bar{P}(s)} \mathrm{d} s+\sigma_{1} B_{1}(t), \\
& \ln \bar{Z}=\ln \bar{Z}_{0}+\left(-b d-\frac{\sigma_{2}^{2}}{2}\right) t+b(1-c) \int_{0}^{t} \frac{\bar{P}(s)}{m+\bar{P}(s)} \mathrm{d} s+\sigma_{2} B_{2}(t) .
\end{aligned}
$$

Hence, we can derive

$$
\begin{aligned}
& \ln \bar{P} \leq \ln \bar{P}_{0}+\left(r-\frac{\sigma_{1}^{2}}{2}\right) t+\sigma_{1} B_{1}(t) \\
& \ln \bar{Z} \leq \ln \bar{Z}_{0}+\left(b-b d-\frac{\sigma_{2}^{2}}{2}\right) t+\sigma_{2} B_{2}(t)
\end{aligned}
$$

Dividing $t$ on the both sides of (16) and letting $t \rightarrow \infty$, we obtain

$$
\begin{aligned}
\limsup _{t \rightarrow \infty} \frac{\ln P}{t} \leq \limsup _{t \rightarrow \infty} \frac{\ln \bar{P}}{t} \leq r-\frac{\sigma_{1}^{2}}{2} \\
\limsup _{t \rightarrow \infty} \frac{\ln Z}{t} \leq \limsup _{t \rightarrow \infty} \frac{\ln \bar{Z}}{t} \leq b-b d-\frac{\sigma_{2}^{2}}{2} \quad \text { a.s. }
\end{aligned}
$$

as required.

Corollary 2.1 Assume $r-\sigma_{1}^{2} / 2<0$ and $b-b d-\sigma_{2}^{2} / 2<0$ hold, then for any initial value $\left(P_{0}, Z_{0}\right) \in R_{+}^{2}$, the solution $(P(t), Z(t))$ to model (2) will be extinct.

\section{Numerical analysis}

In order to facilitate the interpretation of our mathematical results in the stochastic model (2), we proceed to investigate them by numerical simulations.

When choosing the environmental carrying capacity $K=0.7$, under different noise intensities $\sigma_{1}^{2}$ and $\sigma_{2}^{2}$, Figure 3 shows time-series plots of the toxin-producing phytoplankton population $P$ and zooplankton population $Z$ of model (2) with initial value $\left(P_{0}, Z_{0}\right)=(0.45,0.6)$ and the other parameters values are

$$
r=4, \quad m=0.5, \quad a=0.9, \quad b=0.7, \quad c=0.1, \quad d=0.2 \text {. }
$$


(A)

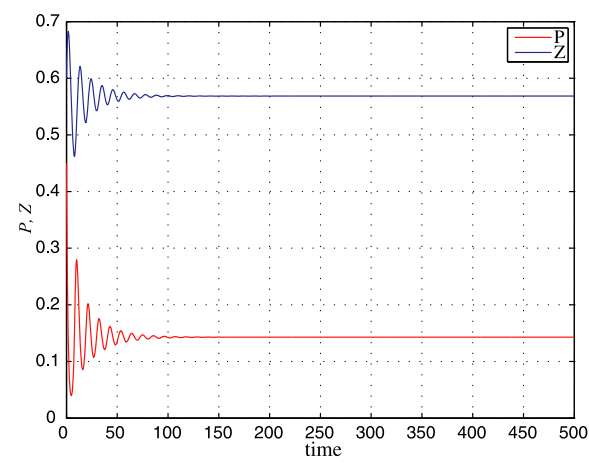

(C)

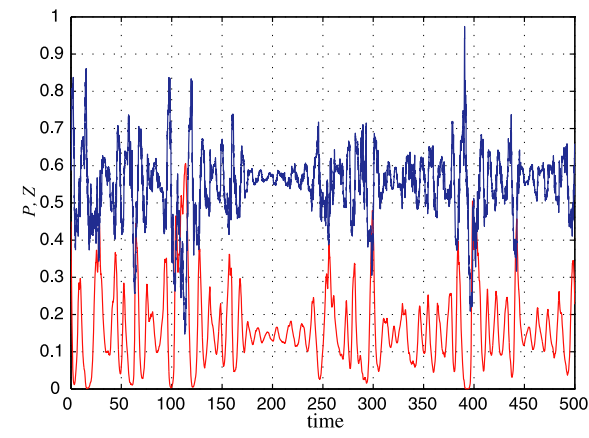

(B)

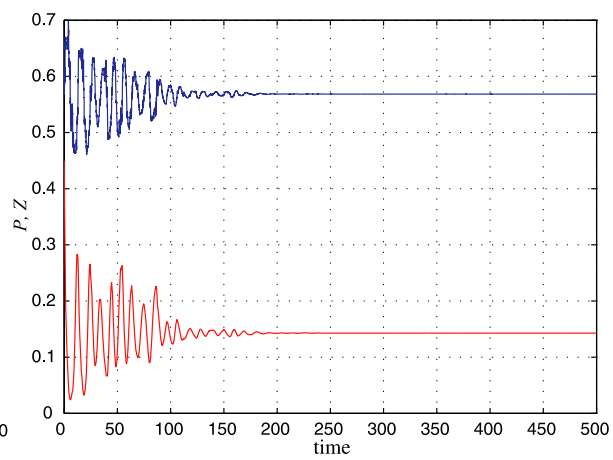

(D)

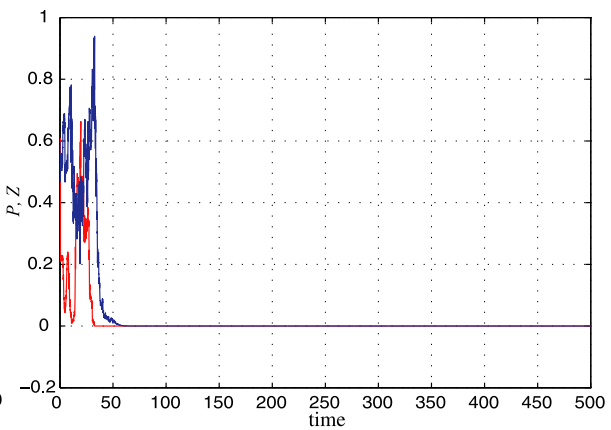

Figure 3 Time-series plots of model (2) with initial value $\left(P_{0}, Z_{0}\right)=(0.45,0.6)$ and different noise intensities $\boldsymbol{\sigma}_{1}^{2}$ and $\boldsymbol{\sigma}_{2}^{2}$. Here $K=0.7$ and other parameters values are taken as (17). (A) $\sigma_{1}^{2}=\sigma_{2}^{2}=0$; (B) $\sigma_{1}^{2}=0.1, \sigma_{2}^{2}=0.25 ;(\mathbf{C}) \sigma_{1}^{2}=0.96, \sigma_{2}^{2}=1.05 ;$ (D) $\sigma_{1}^{2}=8.45, \sigma_{2}^{2}=4.5$.

In Figure 3(A), without noise (i.e., $\left.\sigma_{1}^{2}=\sigma_{2}^{2}=0\right)$, the equilibrium point $\left(P^{*}, Z^{*}\right)=(0.1429$, 0.5685 ) of model (2) is globally asymptotically stable, which illustrates the stable population distribution of both species. In Figure 3(B), with noise intensities $\sigma_{1}^{2}=0.1$ and $\sigma_{2}^{2}=0.25$ satisfying the conditions of Theorem 2.9 , one can see that the random white noise leads to a slight oscillations, and the later random noise makes the oscillations decay, the model ending with the time-independent stability. Comparing Figures 3(A) and (B), one can realize that, if the white noise is not strong, the equilibrium $E^{*}=\left(P^{*}, Z^{*}\right)$ is stochastic asymptotically stable and the stochastic perturbation does not cause sharp changes of the dynamics of model (2). In this case, we get a stable population distribution. In Figure $3(C)$, choosing $\sigma_{1}^{2}=0.96$ and $\sigma_{2}^{2}=1.05$, one can see that as the intensities of the noise are increasing, the phytoplankton and zooplankton population give rise to drastically ruleless oscillations with time. In Figure 3(D), increasing $\sigma_{1}^{2}$ and $\sigma_{2}^{2}$ to 8.45 and 4.5, respectively, which satisfy the conditions $r<\sigma_{1}^{2} / 2$ and $b-b d<\sigma_{2}^{2} / 2$ in Corollary 2.1, we find that model (2) is not permanent and the toxic-phytoplankton population $P$ and zooplankton population $Z$ will go to extinction. In other words, if the conditions $r-\sigma_{1}^{2} / 2<0$ and $b-b d-\sigma_{2}^{2} / 2<0$ hold in Corollary 2.1, the positive equilibrium $\left(P^{*}, Z^{*}\right)$ is no longer globally stable. This shows that large white noise will force the population to become extinct while the population may be persistent under relatively small white noise.

Increasing $K$ to $K=1.2$, we take the other parameters the same as in Figure 3 for model (2) with different noise intensities $\sigma_{1}^{2}$ and $\sigma_{2}^{2}$. Figure 4 illustrates time-series plots and phase portraits of the two species of model (2). In Figure 4(A), without noise (i.e., 


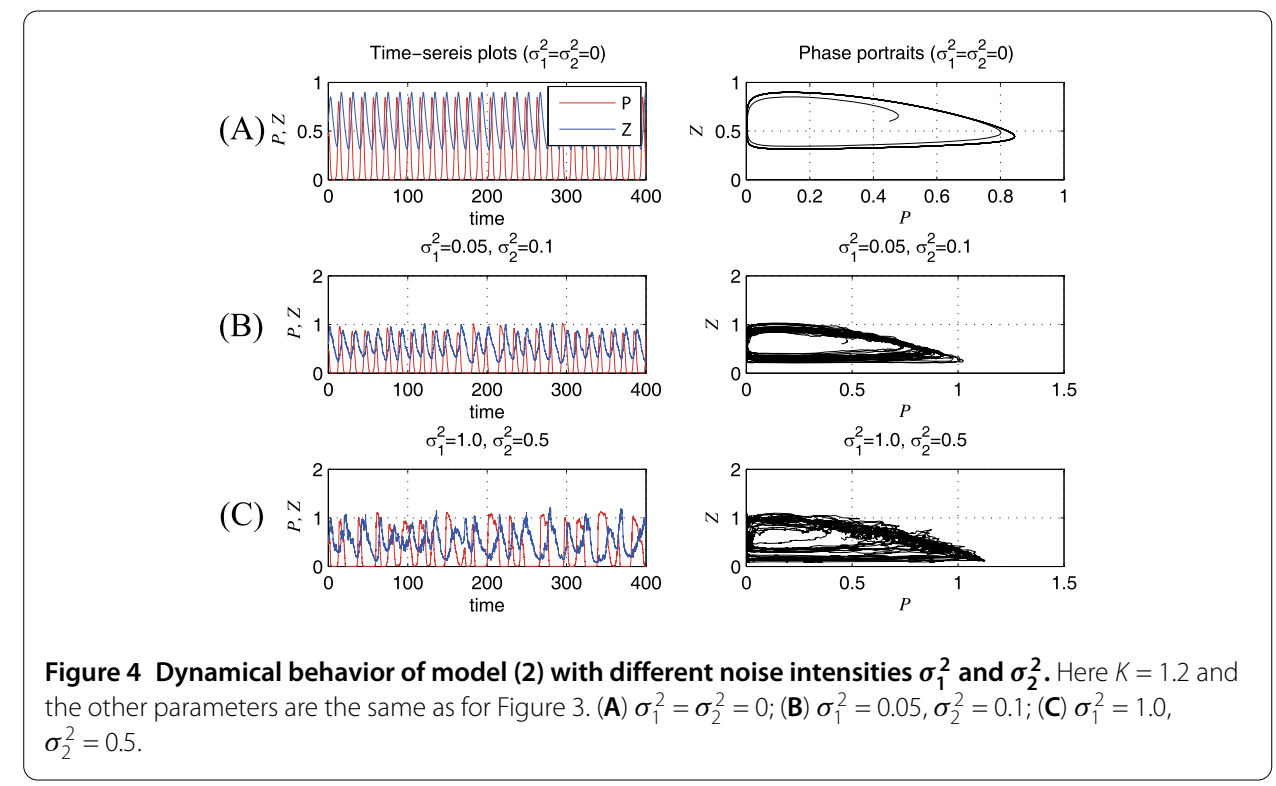

$\sigma_{1}^{2}=\sigma_{2}^{2}=0$ ), a Hopf bifurcation takes place, and a Hopf-bifurcating periodic solution $\left(P^{*}, Z^{*}\right)=(0.1429,0.6293)$ exists and is showed in it. In Figure $4(\mathrm{~B})$ and $(\mathrm{C})$, as the environmental noise intensities $\sigma_{1}^{2}$ and $\sigma_{2}^{2}$ are increased, there is a drastic influence on the dynamical behavior of model (2). In Figure 4(B), $\sigma_{1}^{2}=0.05, \sigma_{2}^{2}=0.1$, and Figure 4(C), $\sigma_{1}^{2}=1.0, \sigma_{2}^{2}=0.5$, which all satisfy the conditions of Theorem 2.9 , one can see that with the perturbation of environmental noise the periodic solution occurs with random periodic variance for model (2). This means that under some conditions and the noise not being strong, stochastic perturbation does not change the permanence of the deterministic plankton model.

We note that, if a positive equilibrium of the deterministic model is globally stable, then the stochastic model preserves the property of the stochastic asymptotical stability when noise is not sufficiently large. In this case, we can ignore the noise and use the deterministic model to describe the population dynamics. However, when the intensity of noise is sufficiently large, the noise can force the population to give rise to drastically ruleless oscillations and to become extinct. In this case, we cannot ignore the effect of noise and only use the stochastic model to describe the population dynamics.

\section{Conclusions and remarks}

In reality, the varying environment is always fraught with all kinds of randomness, but the knowledge as regards the effects of environmental noise on the toxin-producing phytoplankton population and zooplankton population is limited. In this paper, we consider a stochastic toxic-phytoplankton-zooplankton model with Holling type-II functional response. The value of this study lies in two aspects. First, stability criteria of model (1) are analyzed both from a local and a global point of view. The existence of a Hopf bifurcation around an interior equilibrium is established. Second, it presents the complex dynamics of the plankton model (2) with the effect of environmental noise.

For the toxin-producing phytoplankton-zooplankton model (1), by analyzing the corresponding characteristic equation and constructing a Lyapunov function, we study both the local and the global stability of a positive equilibrium. Taking the carrying capacity $K$ 
as the bifurcation parameter, when $K$ crosses a threshold value $K_{H}=m(1-c+d) /(1-c-d)$ the toxin-producing plankton model enters into a Hopf bifurcation and has a periodic orbit around the coexisting equilibrium $E^{*}=\left(P^{*}, Z^{*}\right)$.

In order to study the stochastic model (2), we perturb the deterministic plankton model with respect to environmental noise around the growth rates of toxic-phytoplankton and zooplankton. Applying a Lyapunov function, we show that there is a unique positive solution to the model for any positive initial value. By Itô's formula, we derive that the solution is stochastically bounded and stochastically permanent under some conditions. These conditions depend on the intensities of noise, $\sigma_{1}^{2}$ and $\sigma_{2}^{2}$. When the intensities of noise satisfy some conditions and are not sufficiently large, the population of the stochastic model may be stochastically permanent. Our complete analysis of the model will give some suggestions for the studies on the population dynamics of other models.

Competing interests

The author declares that she has no competing interests.

Author's contributions

The author drafted the manuscript, read and approved the final manuscript.

\section{Acknowledgements}

The author would like to express her gratitude to the editor and referees for their careful reading of the manuscript and a number of excellent criticisms and suggestions.

Received: 21 August 2013 Accepted: 11 December 2013 Published: 16 Jan 2014

\section{References}

1. Medvinsky, AB, Petrovskii, SV, Tikhonova, IA, Malchow, H, Li, BL: Spatiotemporal complexity of plankton and fish dynamics. SIAM Rev. 44, 311-370 (2002)

2. Saha, T, Bandyopadhyay, M: Dynamical analysis of toxin producing phytoplankton-zooplankton interactions. Nonlinear Anal., Real World Appl. 10, 314-332 (2009)

3. Lv, YF, Pei, YZ, Gao, SJ, Li, CG: Harvesting of a phytoplankton-zooplankton model. Nonlinear Anal., Real World Appl. 11, 3608-3619 (2010)

4. Smayda, TJ: Novel and nuisance phytoplankton blooms in the sea: evidence for a global epidemic. In: Toxic Marine Phytoplankton, pp. 29-40. Elsevier, New York (1990)

5. Kirk, KL, Gilbert, JJ: Variations in herbivore response to chemical defenses: zooplankton foraging on toxic cyanobacteria. Ecology 73, 2208-2217 (1992)

6. Hallegraeff, GM: A review of harmful algae blooms and the apparent global increase. Phycologia 32, 79-99 (1993)

7. Bandyopadhyay, M, Saha, T, Pal, R: Deterministic and stochastic analysis of a delayed allelopathic phytoplankton model within fluctuating environment. Nonlinear Anal. Hybrid Syst. 2, 958-970 (2008)

8. Chattopadhyay, J, Sarkar, RR, Mandal, S: Toxin producing plankton may act as a biological control for planktonic blooms: a field study and mathematical modelling. J. Theor. Biol. 215, 333-344 (2002)

9. Upadhyay, RK, Chattopadhyay, J: Chaos to order: role of toxin producing phytoplanton in aquatic systems. Nonlinear Anal.: Model. Control 10,383-396 (2005)

10. Jang, SJ, Baglama, J, Rick, J: Nutrient-phytoplankton-zooplankton models with a toxin. Math. Comput. Model. 43, 105-118 (2006)

11. Chowdhury, T, Roy, S, Chattopadhyay, J: Modeling migratory grazing of zooplankton on toxic and non-toxic phytoplankton. Appl. Math. Comput. 197, 659-671 (2008)

12. Khare, S, Misra, OP, Dhar, J: Role of toxin producing phytoplankton on a plankton ecosystem. Nonlinear Anal. Hybrid Syst. 4, 496-502 (2010)

13. Gakkhar, S, Singh, A: A delay model for viral infection in toxin producing phytoplankton and zooplankton system. Commun. Nonlinear Sci. Numer. Simul. 15, 3607-3620 (2010)

14. Rao, F: Spatiotemporal dynamics in a reaction-diffusion toxic-phytoplankton-zooplankton model. J. Stat. Mech. Theory Exp. 2013, 08014 (2013)

15. Gard, TC: Persistence in stochastic food web models. Bull. Math. Biol. 46, 357-370 (1984)

16. Gard, TC: Stability for multispecies population models in random environments. Nonlinear Anal., Theory Methods Appl. 10, 1411-1419(1986)

17. May, RM: Stability and Complexity in Model Ecosystems. Princeton University Press, New Jersey (2001)

18. Beddington, JR, May, RM: Harvesting natural populations in a randomly fluctuating environment. Science 197 , 463-465 (1977)

19. Mao, XR: Stochastic stabilisation and destabilisation. Syst. Control Lett. 23, 279-290 (1994)

20. Braumann, CA: Variable effort fishing models in random environments. Math. Biosci. 156, 1-19 (1999)

21. Braumann, CA: Variable effort harvesting models in random environments: generalization to density-dependent noise intensities. Math. Biosci. 177, 229-245 (2002)

22. Rudnicki, R, Pichór, K: Influence of stochastic perturbation on prey-predator systems. Math. Biosci. 206, 108-119 (2007) 
23. Liu, QX, Jin, Z, Li, BL: Resonance and frequency-locking phenomena in spatially extended phytoplankton-zooplankton system with additive noise and periodic forces. J. Stat. Mech. Theory Exp. 2008, 05011 (2008)

24. Mukhopadhyay, B, Bhattacharyya, R: Role of gestation delay in a plankton-fish model under stochastic fluctuations. Math. Biosci. 215, 26-34 (2008)

25. LV, JL, Wang, K: Optimal harvest of a stochastic predator-prey model. Adv. Differ. Equ. 2011, 312465 (2011)

26. Li, S, Zhang, X: Dynamics of a stochastic non-autonomous predator-prey system with Beddington-DeAngelis functional response. Adv. Differ. Equ. 2013, 1-20 (2013)

27. Mao, XR: Stochastic Differential Equations and Their Applications. Horwood, Berlin (1997)

28. Murray, JD: Mathematical Biology. Springer, New York (2003)

29. Mao, XR, Yuan, CG, Zou, JZ: Stochastic differential delay equations of population dynamics. J. Math. Anal. Appl. 304, 296-320 (2005)

30. Li, XY, Mao, XR: Population dynamical behavior of non-autonomous Lotka-Volterra competitive system with random perturbation. Discrete Contin. Dyn. Syst. 24, 523-545 (2009)

31. LV, JL, Wang, K: Asymptotic properties of a stochastic predator-prey system with Holling II functional response. Commun. Nonlinear Sci. Numer. Simul. 16, 4037-4048 (2011)

32. Jiang, DQ, Shi, NZ, Li, XY: Global stability and stochastic permanence of a non-autonomous logistic equation with random perturbation. J. Math. Anal. Appl. 340, 588-597 (2008)

\section{Submit your manuscript to a SpringerOpen ${ }^{\circ}$ journal and benefit from:}

- Convenient online submission

Rigorous peer review

- Immediate publication on acceptance

- Open access: articles freely available online

- High visibility within the field

- Retaining the copyright to your article 